\title{
LIMITING BEHAVIOURS OF NON-OSCILLATORY SOLUTIONS OF A PAIR OF COUPLED NONLINEAR DIFFERENTIAL EQUATIONS
}

\author{
WAN-TONG LI ${ }^{1}$ AND SUI SUN CHENG ${ }^{2}$ \\ ${ }^{1}$ Department of Mathematics, Lanzhou University, Lanzhou, \\ Gansu 730000, People's Republic of China \\ ${ }^{2}$ Department of Mathematics, Tsing Hua University, Hsinchu, \\ Taiwan 30043, Republic of China
}

(Received 19 May 1999)

\begin{abstract}
A pair of coupled nonlinear differential equations is studied and asymptotic properties of its non-oscillatory solutions are obtained. In particular, we provide classification schemes for these solutions which are justified by existence criteria.
\end{abstract}

Keywords: coupled differential equation; non-oscillatory solution; classification scheme;

Schauder's Fixed Point Theorem; Knaster's Fixed Point Theorem

AMS 1991 Mathematics subject classification: Primary 34C10; 34C15

\section{Introduction}

A number of oscillation criteria have already been derived for pairs of coupled differential equations of the form

$$
\left.\begin{array}{l}
x^{\prime}(t)=a(t) f(y(t)) \\
y^{\prime}(t)=-b(t) g(x(t))
\end{array}\right\}
$$

(see, for example, Kordonis and Philos [2], Kwong and Wong [5], Morzov [8-10]). Since non-oscillation theorems do not seem to be available, we will provide several such theorems. More specifically, we will classify the non-oscillatory solutions according to their limiting behaviours and then provide necessary and/or sufficient conditions for their existence.

We will assume that

(H1) $a(t)$ and $b(t)$ are non-trivial, non-negative and continuous functions defined on an interval $\left[t_{0}, \infty\right)$; and

(H2) $f(u)$ and $g(u)$ are real, non-decreasing and continuously differentiable functions defined on $R$ such that $x f(x)>0$ and $x g(x)>0$ for $x \neq 0$. 
We will also restrict our attention to those solutions of the differential system (1.1) that exist on some ray $\left[T_{0}, \infty\right)$, where $T_{0} \geqslant t_{0}$ may depend on the particular solution involved. Note that under quite general conditions there will always exist solutions that are continuable to an interval of the form $\left[T_{0}, \infty\right)$, even though there will also exist non-continuable solutions [2].

As usual, a continuous real-valued function defined on an interval $\left[T_{0}, \infty\right)$ is said to be oscillatory if it has arbitrary large zeros, otherwise it is said to be non-oscillatory. A solution $\{x(t), y(t)\}$ of the system (1.1) will be called oscillatory if both $x(t)$ and $y(t)$ are oscillatory, otherwise it is said to be non-oscillatory.

Non-oscillation criteria for solutions of (1.1) are interesting for many reasons. In particular, since several standard second-order ordinary differential equations such as

$$
\begin{aligned}
x^{\prime \prime}(t)+b(t) g(x(t)) & =0 \\
x^{\prime \prime}(t)+b(t)|x(t)|^{\lambda} \operatorname{sign} x(t) & =0 \\
\left(\frac{x^{\prime}(t)}{a(t)}\right)^{\prime}+b(t) g(x(t)) & =0
\end{aligned}
$$

can be written in the form (1.1), these criteria will also provide information on the asymptotic behaviours of these equations. Indeed, we will see that our criteria have contact with those of Kusano and Singh [5], Kusano and Naito [3], $\mathrm{Li}$ [6], Lu [7], Naito [11-13], Ruan [14] and others who deal with these equations.

System (1.1) is naturally classified into four classes according to whether

$$
\int_{t_{0}}^{\infty} a(s) \mathrm{d} s<\infty, \quad \int_{t_{0}}^{\infty} a(s) \mathrm{d} s=\infty, \quad \int_{t_{0}}^{\infty} b(s) \mathrm{d} s<\infty \quad \text { or } \quad \int_{t_{0}}^{\infty} b(s) \mathrm{d} s=\infty .
$$

By symmetry considerations, we will, however, restrict our attention to the cases where

$$
\int_{t_{0}}^{\infty} a(s) \mathrm{d} s<\infty \text { or } \int_{t_{0}}^{\infty} a(s) \mathrm{d} s=\infty
$$

For this reason, we will employ the following notation:

$$
A(s)=\int_{s}^{\infty} a(u) \mathrm{d} u, \quad s \geqslant t_{0}
$$

and

$$
A(s, t)=\int_{s}^{t} a(u) \mathrm{d} u, \quad t_{0} \leqslant s \leqslant t
$$

Lemma 1.1. Suppose conditions (H1) and (H2) hold. Suppose further that the function $a(t)$ is not identically zero on any interval of the form $\left[\tau_{0}, \infty\right)$, where $\tau_{0} \geqslant t_{0}$, then the component function $x(t)$ of a non-oscillatory solution $\{x(t), y(t)\}$ of (1.1) is also non-oscillatory. 
For the proof, assume to the contrary that $x(t)$ is oscillatory but that $y(t)$ is eventually positive, then, in view of $(1.1), x^{\prime}(t)=a(t) f(y(t)) \geqslant 0$ for all large $t$, and $x^{\prime}\left(t_{i}\right)=$ $a\left(t_{i}\right) f\left(y\left(t_{i}\right)\right)>0$ for an increasing and divergent sequence $\left\{t_{i}\right\}$. Thus, $x(t)>0$ for all large $t$, or $x(t)<0$ for all large $t$. This is a contradiction. The case where $y(t)$ is eventually negative is similarly proved.

Similarly, if $b(t)$ is not identically zero on any interval of the form $\left[\tau_{0}, \infty\right)$, then the component function $y(t)$ of a non-oscillatory solution $\{x(t), y(t)\}$ is also non-oscillatory. Therefore, under the additional condition

(H3) $a(t)$ and $b(t)$ are not identically zero on any interval of the form $\left[\tau_{0}, \infty\right)$, where $\tau_{0} \geqslant t_{0}$,

each component function of a non-oscillatory solution $\{x(t), y(t)\}$ of $(1.1)$ is eventually of one sign.

If we now interpret (1.1) as a (time-varying) vector field in the plane and its solutions as trajectories, then we see that each non-oscillatory solution corresponds to a trajectory which ultimately lies in one of the four open quadrants of the plane. In view of the directions of the vector field in each open quadrant, it is also clear that the component functions of a non-oscillatory trajectory must be monotone.

\section{The case $A\left(t_{0}\right)=\infty$}

We now impose an additional condition on (1.1), namely that $A\left(t_{0}\right)=\infty$. We assert that any non-oscillatory solution $\{x(t), y(t)\}$ of (1.1) must ultimately lie in the first or the third open quadrant.

Lemma 2.1. Suppose conditions (H1)-(H3) hold. Suppose further that $A\left(t_{0}\right)=\infty$. Then any non-oscillatory trajectory $\{x(t), y(t)\}$ of (1.1) must ultimately lie in the first or the third open quadrant and the function $y(t)$ must converge. Furthermore, there exist $c_{1}>0, c_{2}>0$ and $T \geqslant t_{0}$ such that $c_{1} \leqslant x(t) \leqslant c_{2} A\left(t_{0}, t\right)$, or $-c_{2} A\left(t_{0}, t\right) \leqslant x(t) \leqslant c_{1}$ for $t \geqslant T$.

Proof. Assume without loss of generality that $x(t)>0$ and $y(t)>0$, or $x(t)>0$ and $y(t)<0$ for $t \geqslant T_{0}$. The latter case cannot happen. Otherwise, $x^{\prime}(t) \leqslant 0$ and $y^{\prime}(t) \leqslant 0$ for $t \geqslant T_{0}$. Hence,

$$
x^{\prime}(t)=a(t) f(y(t)) \leqslant a(t) f\left(y\left(T_{0}\right)\right), \quad t \geqslant T_{0}
$$

which implies

$$
0<x(t) \leqslant x\left(T_{0}\right)+f\left(y\left(T_{0}\right)\right) \int_{T_{0}}^{t} a(s) \mathrm{d} s=x\left(T_{0}\right)+f\left(y\left(T_{0}\right)\right) A\left(T_{0}, t\right) \rightarrow-\infty,
$$

a contradiction. Thus, $x(t)>0, y(t)>0, x^{\prime}(t) \geqslant 0$ and $y^{\prime}(t) \leqslant 0$ for $t \geqslant T_{0}$. It follows that $y(t)$ monotonically decreases to a non-negative constant, and $x(t)$ monotonically increases. Furthermore, since (2.1) holds in this case, we see that

$$
0<x\left(T_{0}\right) \leqslant x(t) \leqslant x\left(T_{0}\right)+f\left(y\left(T_{0}\right)\right) A\left(T_{0}, t\right) \leqslant c_{2} A\left(t_{0}, t\right), \quad t \geqslant T_{0},
$$

for some $c_{2}>0$ since $A\left(t_{0}\right)=\infty$. The proof is complete. 
We have shown that a non-oscillatory solution $\{x(t), y(t)\}$ must ultimately lie in the first or the third quadrant, and that the function $y(t)$ must converge. Note that since $x^{\prime}(t) \geqslant 0$ or $x^{\prime}(t) \leqslant 0$ for all large $t$, we see further that $x(t)$ either converges to some nonzero constant or diverges to positive infinity or to negative infinity as $t \rightarrow \infty$. However, if $x(t)$ converges to some non-zero constant, then $y(t)$ must converge to 0 . Indeed, if $\lim _{t \rightarrow \infty} y(t)=d>0$, then since

$$
x^{\prime}(t)=a(t) f(y(t)) \geqslant a(t) f(d)
$$

for all large $t$, we have

$$
x(t) \geqslant x(M)+f(d) \int_{M}^{t} a(s) \mathrm{d} s \rightarrow \infty
$$

which is a contradiction.

In view of the above considerations, we may now make the following classification. Let $\Omega$ be the set of all non-oscillatory solutions of (1.1) and $\Omega^{+}$be the subset of $\Omega$ containing those which ultimately lie in the first open quadrant. Suppose conditions (H1)-(H3) hold and $A\left(t_{0}\right)=\infty$. Then any non-oscillatory solution in $\Omega^{+}$must belong to one of the following three classes:

$$
\begin{aligned}
\Omega^{+}(+, 0) & =\left\{\{x, y\} \in \Omega^{+} \mid \lim _{t \rightarrow \infty} x(t) \in(0, \infty), \lim _{t \rightarrow \infty} y(t)=0\right\} \\
\Omega^{+}(\infty, 0) & =\left\{\{x, y\} \in \Omega^{+} \mid \lim _{t \rightarrow \infty} x(t)=\infty, \lim _{t \rightarrow \infty} y(t)=0\right\}, \\
\Omega^{+}(\infty,+) & =\left\{\{x, y\} \in \Omega^{+} \mid \lim _{t \rightarrow \infty} x(t)=\infty, \lim _{t \rightarrow \infty} y(t) \in(0, \infty)\right\}
\end{aligned}
$$

A similar classification is also available for non-oscillatory solutions that lie, ultimately, in the third open quadrant.

In order to further justify our classification scheme, we derive several necessary and sufficient conditions for the existence of each type of non-oscillatory solutions.

Theorem 2.2. Suppose conditions (H1)-(H3) hold and $A\left(t_{0}\right)=\infty$. A necessary and sufficient condition for (1.1) to have a non-oscillatory solution $\{x(t), y(t)\}$ which belongs to $\Omega^{+}(+, 0)$ is that

$$
\int_{t_{0}}^{\infty} a(t)\left|f\left(\int_{t}^{\infty} b(s) g(c) \mathrm{d} s\right)\right| \mathrm{d} t<\infty
$$

for some $c>0$.

Proof. Let $\{x(t), y(t)\}$ be a solution in $\Omega^{+}(+, 0)$ such that $\lim _{t \rightarrow \infty} x(t)=\alpha>0$. Then there exist $c_{1}>0$ and $T$ such that $c_{1} \leqslant x(t)$ for $t \geqslant T$. In view of (1.1),

$$
y(t)=\int_{t}^{\infty} b(s) g(x(s)) \mathrm{d} s, \quad t \geqslant T
$$


and

$$
\begin{aligned}
\infty & >\alpha-x(T)=\int_{T}^{\infty} a(s) f(y(s)) \mathrm{d} s \\
& =\int_{T}^{\infty} a(s) f\left(\int_{s}^{\infty} b(u) g(x(u)) \mathrm{d} u\right) \mathrm{d} s \\
& \geqslant \int_{T}^{\infty} a(s) f\left(\int_{s}^{\infty} b(u) g\left(c_{1}\right) \mathrm{d} u\right) \mathrm{d} s .
\end{aligned}
$$

Conversely, choose a number $M$ so large that

$$
\int_{M}^{\infty} a(s) f\left(\int_{s}^{\infty} b(u) g(c) \mathrm{d} u\right) \mathrm{d} s<c / 2 .
$$

Let $X$ be the set of all bounded continuous real-valued functions $x=x(t)$ defined on $[M, \infty)$ with norm $\|x\|=\sup _{t \geqslant M}|x(t)|$. Let $\Psi$ be the subset of the Banach space $X$ defined by

$$
\Psi=\{x(t) \in X \mid c / 2<x(t) \leqslant c, t \geqslant T\} .
$$

Then $\Psi$ is a bounded, convex and closed subset of $X$. Let us define an operator $F: \Psi \rightarrow X$ as follows:

$$
(F x)(t)=c-\int_{t}^{\infty} a(s) f\left(\int_{s}^{\infty} b(u) g(x(u)) \mathrm{d} u\right) \mathrm{d} s, t \geqslant M .
$$

The mapping $F$ has the following properties. First of all, $F$ maps $\Psi$ into $\Psi$. Indeed, if $x \in \Psi$, then

$$
\begin{gathered}
c \geqslant(F x)(t)=c-\int_{t}^{\infty} a(s) f\left(\int_{s}^{\infty} b(u) g(x(u)) \mathrm{d} u\right) \mathrm{d} s \\
\geqslant c-\int_{M}^{\infty} a(s) f\left(\int_{s}^{\infty} b(u) g(x(c)) \mathrm{d} u\right) \mathrm{d} s \geqslant c / 2 .
\end{gathered}
$$

Next, we show that $F$ is continuous. Let $\left\{x_{i}\right\}$ be a convergent sequence of functions in $\Psi$ such that $\lim _{i \rightarrow \infty}\left\|x_{i}-x\right\|=0$. Since $\Psi$ is closed, $x \in \Psi$. By the definition of $F$, we have

$$
\begin{aligned}
\mid\left(F x_{i}\right)(t) & -(F x)(t) \mid \\
& \leqslant \int_{t}^{\infty} a(s)\left|f\left(\int_{s}^{\infty} b(u) g\left(x_{i}(u)\right) \mathrm{d} u\right) \mathrm{d} s-f\left(\int_{s}^{\infty} b(u) g(x(u)) \mathrm{d} u\right)\right| \mathrm{d} s .
\end{aligned}
$$

By the continuity of $f$ and $g$ and Lebesgue's Dominated Convergence Theorem, it follows that $\lim _{i \rightarrow \infty}\left\|F x_{i}-F x\right\|=0$.

Finally, we show that $F \Psi$ is precompact. Let $x \in \Psi$ and $s, t \geqslant M$. Then, assuming, without loss of generality, that $s>t$,

$$
\begin{aligned}
|(F x)(s)-(F x)(t)| & =\left|\int_{t}^{s} a(u) f\left(\int_{u}^{\infty} b(v) g(x(v)) \mathrm{d} v\right) \mathrm{d} u\right| \\
& \leqslant \int_{t}^{s} a(u) f\left(\int_{u}^{\infty} b(v) g(c) \mathrm{d} v\right) \mathrm{d} u .
\end{aligned}
$$


In view of (2.2), for any $\varepsilon>0$, there exists a $\delta>0$ such that $|s-t|<\delta$, thus we have

$$
|(F x)(s)-(F x)(t)|<\varepsilon .
$$

This means that $F \Psi$ is precompact.

By Schauder's Fixed Point Theorem, we may conclude that there exists an $x \in \Psi$ such that $x=F x$. Set

$$
y(t)=\int_{t}^{\infty} b(v) g(x(v)) \mathrm{d} v, \quad t \geqslant M,
$$

then $\lim _{t \rightarrow \infty} y(t)=0$ and $y^{\prime}(t)=-b(t) g(x(t))$. On the other hand,

$$
\begin{aligned}
x(t) & =(F x)(t)=c-\int_{t}^{\infty} a(s) f\left(\int_{s}^{\infty} b(u) g(x(u)) \mathrm{d} u\right) \mathrm{d} s \\
& =c-\int_{t}^{\infty} a(s) f(y(s)) \mathrm{d} s,
\end{aligned}
$$

and thus $\lim _{t \rightarrow \infty} x(t)=c$ and $x^{\prime}(t)=a(t) f(y(t))$, as required. The proof is complete.

Theorem 2.3. Suppose conditions $(H 1)-(H 3)$ hold and $A\left(t_{0}\right)=\infty$. A necessary and sufficient condition for (1.1) to have a non-oscillatory solution $\{x(t), y(t)\}$ which belongs to $\Omega^{+}(\infty,+)$ is that

$$
\int_{t_{0}}^{\infty} b(t)\left|g\left(c A\left(t_{0}, s\right)\right)\right| \mathrm{d} t<\infty
$$

for some $c>0$.

Proof. Let $\{x(t), y(t)\}$ be a solution in $\Omega^{+}(\infty,+)$ such that $\lim _{t \rightarrow \infty} y(t)=\beta>0$. Then there exist four positive constants, $c_{1}, c_{2}, d_{1}, d_{2}$, and $T \geqslant t_{0}$ such that

$$
c_{1} \leqslant x(t) \leqslant c_{2} A\left(t_{0}, t\right), \quad d_{1} \leqslant y(t) \leqslant d_{2}
$$

for $t \geqslant T$. In view of (1.1), we have

$$
\begin{aligned}
x(t) & =x\left(t_{0}\right)+\int_{t_{0}}^{t} a(s) f(y(s)) \mathrm{d} s \\
& \geqslant x\left(t_{0}\right)+\int_{t_{0}}^{t} a(s) f\left(d_{1}\right) \mathrm{d} s \\
& \geqslant f\left(d_{1}\right) A\left(t_{0}, t\right) \\
& =c A\left(t_{0}, t\right)
\end{aligned}
$$

and

$$
\infty>y(T)-\beta=\int_{T}^{\infty} b(s) g(x(s)) \mathrm{d} s \geqslant \int_{T}^{\infty} b(s) g\left(c A\left(t_{0}, s\right)\right) \mathrm{d} s .
$$

Conversely, pick a number $T \geqslant t_{0}$ so that

$$
\int_{T}^{\infty} b(s) g(c A(T, s)) \mathrm{d} s<d
$$


where $d=f^{-1}(c) / 2$. Let $X$ be the partly ordered Banach space of all continuous realvalued functions $x(t)$ with the norm

$$
\|x\|=\sup _{t \geqslant T} \frac{|x(t)|}{A(T, t)},
$$

and the usual pointwise ordering. Let $\Psi$ be the subset of $X$ defined by

$$
\Psi=\{x \in X \mid f(d) A(T, t) \leqslant x(t) \leqslant f(2 d) A(T, t), t \geqslant T\} .
$$

For any subset $B$ of $\Psi$, it is obvious that $\inf B \in X$ and $\sup B \in X$. Let us further define an operator $F: \Psi \rightarrow X$ as follows:

$$
(F x)(t)=\int_{T}^{t} a(s) f\left(d+\int_{s}^{\infty} b(u) g(x(u)) \mathrm{d} u\right) \mathrm{d} s, \quad t \geqslant T .
$$

The mapping $F$ satisfies the assumptions of Knaster's Fixed Point Theorem: $F$ maps $\Psi$ into itself and $F$ is non-decreasing. The latter is easy to see. As for the former statement, note that, for any $x \in \Psi$,

$$
(F x)(t) \geqslant A(T, t) f(d), \quad t \geqslant T
$$

and

$$
\begin{aligned}
(F x)(t) & \leqslant \int_{T}^{t} a(s) f\left(d+\int_{T}^{\infty} b(u) g(f(2 a) A(T, u)) \mathrm{d} u\right) \mathrm{d} s \\
& =\int_{T}^{t} a(s) f\left(d+\int_{T}^{\infty} b(u) g(c A(T, u)) \mathrm{d} u\right) \mathrm{d} s \\
& \leqslant f(2 d) A(T, t)
\end{aligned}
$$

for $t \geqslant T$, as desired.

By Knaster's Fixed Point Theorem, we may conclude that there exists an $x \in \Psi$ such that $x=F x$. Set

$$
y(t)=d+\int_{t}^{\infty} b(u) g(x(u)) \mathrm{d} u, \quad t \geqslant T .
$$

Then $\lim _{t \rightarrow \infty} y(t)=d$ and $y^{\prime}(t)=-b(t) g(x(t))$. On the other hand,

$$
x(t)=(F x)(t)=\int_{T}^{t} a(s) f(y(s)) \mathrm{d} s \geqslant f(d) \int_{T}^{t} a(s) \mathrm{d} s=f(d) A(T, t),
$$

so that $\lim _{t \rightarrow \infty} x(t)=\infty$ and $x^{\prime}(t)=a(t) f(y(t))$, as required. The proof is complete.

Finally, we provide a sufficient condition for the existence of a solution in $\Omega^{+}(\infty, 0)$.

Theorem 2.4. Suppose conditions (H1)-(H3) hold and $A\left(t_{0}\right)=\infty$. A sufficient condition for (1.1) to have a non-oscillatory solution $\{x(t), y(t)\}$ in $\Omega^{+}(\infty, 0)$ is that

$$
\int_{t_{0}}^{\infty} b(t)\left|g\left(c A\left(t_{0}, s\right)\right)\right| \mathrm{d} t<\infty
$$


for some $c>0$, and

$$
\int_{t_{0}}^{\infty} a(t)\left|f\left(\int_{t}^{\infty} b(s) g(d) \mathrm{d} s\right)\right| \mathrm{d} t=\infty
$$

for any $d>0$.

Proof. Let $\delta>c$ be fixed. Denote by $C\left[t_{0}, \infty\right)$ the space of all continuous functions on $\left[t_{0}, \infty\right)$ with the topology of uniform convergence on every compact subinterval of $\left[t_{0}, \infty\right)$. Consider the subset $\Psi$ of $C\left[t_{0}, \infty\right)$ consisting of all $x \in C\left[t_{0}, \infty\right)$ such that

$$
\delta \leqslant x(t) \leqslant \delta+\int_{T}^{t} a(s) f\left(\int_{s}^{\infty} b(u) g(x(u)) \mathrm{d} u\right) \mathrm{d} s, \quad t \geqslant T .
$$

Let us define an operator $F: \Psi \rightarrow C\left[t_{0}, \infty\right)$ as follows:

$$
(F x)(t)=\delta+\int_{T}^{t} a(s) f\left(\int_{s}^{\infty} b(u) g(x(u)) \mathrm{d} u\right) \mathrm{d} s, \quad t \geqslant T .
$$

It is clear that $F$ is well defined and maps $\Psi$ into $C\left[t_{0}, \infty\right)$. By reasoning similar to that in the proof of Theorem 2.2, we may also show that $F$ maps $\Psi$ into $\Psi$, that $F$ is continuous on $\Psi$, and that $F \Psi$ is relatively compact. Schauder's Fixed Point Theorem then implies that $F$ has a fixed point $x$ in $\Psi$. Set

$$
y(t)=\int_{t}^{\infty} b(u) g(x(u)) \mathrm{d} u, \quad t \geqslant T .
$$

Then $\lim _{t \rightarrow \infty} y(t)=0$ and $y^{\prime}(t)=-b(t) g(x(t))$. On the other hand,

$$
x(t)=(F x)(t)=\delta+\int_{T}^{t} a(s) f(y(s)) \mathrm{d} s,
$$

so that $x^{\prime}(t)=a(t) f(y(t))$ for $t \geqslant T$. We assert that $\lim _{t \rightarrow \infty} x(t)=\infty$. Indeed, $x(t)$ either converges to some positive limit or diverges to $\infty$. If $\lim _{t \rightarrow \infty} x(t)=d>0$, then $x(s) \geqslant d / 2$ for $s \geqslant S$. In view of (2.6),

$$
\begin{aligned}
x(t) & =\delta+\int_{T}^{t} a(s) f\left(\int_{s}^{\infty} b(u) g(x(u)) \mathrm{d} u\right) \mathrm{d} s \\
& \geqslant \delta+\int_{T}^{t} a(s) f\left(\int_{s}^{\infty} b(u) g(d / 2) \mathrm{d} u\right) \mathrm{d} s=\infty,
\end{aligned}
$$

which is a contradiction.

\section{The case $A\left(t_{0}\right)<\infty$}

We now impose an additional condition on (1.1), namely that $A\left(t_{0}\right)<\infty$. We assert that the trajectory of any non-oscillatory solution $\{x(t), y(t)\}$ of $(1.1)$ approaches some vertical line as $t \rightarrow \infty$. 
Lemma 3.1. Suppose conditions (H1)-(H3) hold. Suppose further that $A\left(t_{0}\right)<\infty$. Then any non-oscillatory trajectory $\{x(t), y(t)\}$ of (1.1) tends to some vertical line $x=\alpha$ as $t \rightarrow \infty$. Furthermore, there exist $c_{1}, c_{2}>0$ and $T \geqslant t_{0}$ such that $c_{1} A(t) \leqslant x(t) \leqslant c_{2}$, or $-c_{2} \leqslant x(t) \leqslant-c_{1} A(t)$ for $t \geqslant T$.

Proof. Without loss of generality, suppose $x(t)>0$ and $y(t)>0$ for $t \geqslant T_{0}$, or $x(t)>0$ and $y(t)<0$ for $t \geqslant T_{0}$. If the former case holds, then in view of $(1.1), x^{\prime}(t) \geqslant 0$ and $y^{\prime}(t) \leqslant 0$ for $t \geqslant T_{0}$. Thus,

$$
0 \leqslant x^{\prime}(t)=a(t) f(y(t)) \leqslant a(t) f\left(y\left(T_{0}\right)\right), \quad t \geqslant T_{0},
$$

which implies that $x(t)$ monotonically increases to a non-negative constant $\alpha$, since

$$
x(t) \leqslant x\left(T_{0}\right)+f\left(y\left(T_{0}\right)\right) \int_{T_{0}}^{t} a(s) \mathrm{d} s \leqslant x\left(T_{0}\right)+f\left(y\left(T_{0}\right)\right) A\left(t_{0}\right)<\infty .
$$

Furthermore, it is clear that $A(t) \leqslant x(t)<\alpha+1$ for all large $t$, since $A(t) \rightarrow 0$ as $t \rightarrow \infty$.

Suppose the later case holds. Then in view of (1.1), we see that $x^{\prime}(t) \leqslant 0$ and $y^{\prime}(t) \leqslant 0$ for $t \geqslant T_{0}$. Hence, $x(t)$ monotonically decreases to a non-negative constant $\beta$. Since

$$
x^{\prime}(t)=a(t) f(y(t)) \leqslant a(t) f\left(y\left(T_{0}\right)\right), \quad t \geqslant T_{0}
$$

thus

$$
0 \leqslant \beta=x(s)+f\left(y\left(T_{0}\right)\right) \int_{s}^{\infty} a(u) \mathrm{d} u=x(s)+f\left(y\left(T_{0}\right)\right) A(s), \quad t \geqslant T_{0},
$$

we see further that $\beta+1 \geqslant x(s) \geqslant-f\left(y\left(T_{0}\right)\right) A(s)$ for all large $s$. The proof is complete.

We have shown that a non-oscillatory solution $\{x(t), y(t)\}$ must ultimately lie in one of the four open quadrants of the plane. In the case where $\{x(t), y(t)\}$ is eventually in the first open quadrant, then in view of our previous lemmas, $x(t)$ monotonically increases and approaches a positive constant and $y(t)$ decreases and approaches a nonnegative constant. In the case where $\{x(t), y(t)\}$ is eventually in the fourth quadrant, then $x(t)$ decreases and converges to a non-negative constant and $y(t)$ decreases and either converges to a negative constant or diverges to $-\infty$. The other two cases can similarly be analysed.

In view of the above considerations, we may now make the following classification. Let $\Omega$ be the set of all non-oscillatory solutions of (1.1) and $\Omega_{++}, \Omega_{+-}$be, respectively, the subsets of $\Omega$ containing those which ultimately lie in the first or the fourth open quadrant. Suppose conditions (H1)-(H3) hold and $A\left(t_{0}\right)<\infty$. Then any solution in $\Omega_{++}$must belong to the following classes

$$
\begin{aligned}
& \Omega_{++}(+,+)=\left\{\{x, y\} \in \Omega_{++} \mid \lim _{t \rightarrow \infty} x(t) \in(0, \infty), \lim _{t \rightarrow \infty} y(t) \in(0, \infty)\right\} \\
& \Omega_{++}(+, 0)=\left\{\{x, y\} \in \Omega_{++} \mid \lim _{t \rightarrow \infty} x(t) \in(0, \infty), \lim _{t \rightarrow \infty} y(t)=0\right\}
\end{aligned}
$$


while any solution in $\Omega_{+-}$must belong to the following classes:

$$
\begin{aligned}
\Omega_{+-}(+,-) & =\left\{\{x, y\} \in \Omega_{+-} \mid \lim _{t \rightarrow \infty} x(t) \in(0, \infty), \lim _{t \rightarrow \infty} y(t) \in(-\infty, 0)\right\}, \\
\Omega_{+-}(+,-\infty) & =\left\{\{x, y\} \in \Omega_{+-} \mid \lim _{t \rightarrow \infty} x(t) \in(0, \infty), \lim _{t \rightarrow \infty} y(t)=-\infty\right\}, \\
\Omega_{+-}(0,-) & =\left\{\{x, y\} \in \Omega_{+-} \mid \lim _{t \rightarrow \infty} x(t)=0, \lim _{t \rightarrow \infty} y(t) \in(-\infty, 0)\right\}, \\
\Omega_{+-}(0,-\infty) & =\left\{\{x, y\} \in \Omega_{+-} \mid \lim _{t \rightarrow \infty} x(t)=0, \lim _{t \rightarrow \infty} y(t)=-\infty\right\} .
\end{aligned}
$$

Again, in order to justify our classification scheme, we derive several necessary and/or sufficient conditions for the existence of each type of non-oscillatory solution.

Theorem 3.2. Suppose conditions (H1)-(H3) hold and $A\left(t_{0}\right)<\infty$. A necessary and sufficient condition for (1.1) to have a non-oscillatory solution in $\Omega_{+-}(0,-)$ is that

$$
\int_{t_{0}}^{\infty} b(t) g(c A(t)) \mathrm{d} t<\infty
$$

for some $c>0$.

Proof. Let $\{x(t), y(t)\}$ be a solution in $\Omega_{+_{-}}(0,-)$ such that $\lim _{t \rightarrow \infty} x(t)=0$ and $\lim _{t \rightarrow \infty} y(t)=\beta<0$. Since $y(t)$ is monotone, there exist two positive constants $c_{1}, c_{2}$ and $T \geqslant t_{0}$ such that $-c_{1} \leqslant y(t) \leqslant-c_{2}$ for $t \geqslant T$. On the other hand, in view of the second equation of (1.1), we have

$$
y(t)=y(T)-\int_{T}^{t} b(s) g(x(s)) \mathrm{d} s .
$$

Since $\lim _{t \rightarrow \infty} y(t)=\beta<0$, then

$$
\int_{T}^{\infty} b(s) g(x(s)) \mathrm{d} s<\infty
$$

Furthermore, we see from Lemma 3.1 that

$$
g(x(t)) \geqslant g(c A(t))
$$

which implies that

$$
\int_{t_{0}}^{\infty} b(t) g(c A(t)) \mathrm{d} t<\infty
$$

Conversely, suppose that (3.1) holds. Then, in view of $A\left(t_{0}\right)<\infty$, there exists $T \geqslant t_{0}$ such that

$$
\int_{T}^{\infty} b(t) g(c A(t)) \mathrm{d} t<d
$$

where $d=f^{-1}(c) / 2$. Let $X$ be the partly ordered Banach space of all continuous realvalued functions $x(t)$ endowed with the norm

$$
\|x\|=\sup _{t \geqslant T}|x(t)|
$$


and with the usual pointwise ordering $\leqslant$. Define a subset $\Omega$ of $X$ as follows:

$$
\Omega=\{x \in X \mid f(d) A(t) \leqslant x(t) \leqslant f(2 d) A(t), t \geqslant T\} .
$$

For any subset $B$ of $\Omega$, it is obvious that inf $B \in \Omega$ and $\sup B \in \Omega$. Let us further define an operator $F: \Omega \rightarrow X$ as follows:

$$
(F x)(t)=\int_{T}^{t} a(s) f\left(d+\int_{s}^{\infty} b(u) g(x(u)) \mathrm{d} u\right) \mathrm{d} s, \quad t \geqslant T .
$$

The mapping $F$ satisfies the assumptions of Knaster's Fixed Point Theorem [1]: $F$ is nondecreasing (since $f$ and $g$ are non-decreasing). It maps $\Omega$ into itself. Indeed, if $x \in \Omega$, then

$$
(F x)(t) \geqslant f(d) A(t), \quad t \geqslant T
$$

and

$$
\begin{aligned}
(F x)(t) & \leqslant \int_{T}^{t} a(s) f\left(d+\int_{T}^{\infty} b(u) g(f(2 a) A(u)) \mathrm{d} u\right) \mathrm{d} s \\
& =\int_{T}^{t} a(s) f\left(d+\int_{T}^{\infty} b(u) g(c A(u)) \mathrm{d} u\right) \mathrm{d} s \leqslant f(2 d) A(t), \quad t \geqslant T .
\end{aligned}
$$

By Knaster's Fixed Point Theorem [1], we can conclude that there exists an $x \in \Omega$ such that $x=F x$. Set

$$
y(t)=d+\int_{t}^{\infty} b(u) g(x(u)) \mathrm{d} u, \quad t \geqslant T .
$$

Then

$$
y^{\prime}(t)=-b(t) g(x(t)) \text { and } \quad \lim _{t \rightarrow \infty} y(t)=d
$$

On the other hand,

$$
\begin{aligned}
x(t) & =(F x)(t)=\int_{T}^{t} a(s) f(y(s)) \mathrm{d} s \leqslant f(2 d) \int_{T}^{t} a(s) \mathrm{d} s=f(2 d) A(t), \\
x^{\prime}(t) & =a(t) f(y(t))
\end{aligned}
$$

and in view of the fact that $A(t) \rightarrow 0$ as $t \rightarrow \infty$, we have

$$
\lim _{t \rightarrow \infty} x(t)=0 .
$$

The proof is complete.

Theorem 3.3. Suppose conditions (H1)-(H3) hold and $A\left(t_{0}\right)<\infty$. A necessary and sufficient condition for (1.1) to have a non-oscillatory solution in $\Omega_{++}(+,+)$is that

$$
\int_{T}^{\infty} a(s) f\left(\beta+\int_{s}^{\infty} b(u) g(c) \mathrm{d} u\right) \mathrm{d} s<\infty
$$

for some $c>0$ and $\beta>0$. 
Proof. Let $\{x(t), y(t)\}$ be a solution of (1.1) such that $\lim _{t \rightarrow \infty} x(t)=\alpha>0$ and $\lim _{t \rightarrow \infty} y(t)=\beta>0$. Then there exist two positive constants $c_{1}, c_{2}$ and $T \geqslant t_{0}$ such that $c_{1} \leqslant x(t) \leqslant c_{2}$ for $t \geqslant T$. In view of the first equation of (1.1), we have

$$
x(t)=x(T)+\int_{T}^{t} a(s) f(y(s)) \mathrm{d} s .
$$

Since $\lim _{t \rightarrow \infty} x(t)=\alpha>0$, we have

$$
\int_{T}^{\infty} a(s) f(y(s)) \mathrm{d} s<\infty
$$

Furthermore, we see from the second equation of (1.1) that

$$
y(t)=\beta+\int_{t}^{\infty} b(s) g(x(s)) \mathrm{d} s
$$

and

$$
\beta+\int_{t}^{\infty} b(s) g\left(c_{1}\right) \mathrm{d} s \leqslant y(t) \leqslant \beta+\int_{t}^{\infty} b(s) g\left(c_{2}\right) \mathrm{d} s
$$

Thus,

$$
\int_{T}^{\infty} a(s) f\left(\beta+\int_{s}^{\infty} b(u) g\left(c_{2}\right) \mathrm{d} u\right) \mathrm{d} s<\infty .
$$

Conversely, suppose that (3.3) holds. Choose $T \geqslant t_{0}$ so large that

$$
\int_{T}^{\infty} a(s) f\left(\beta+\int_{s}^{\infty} b(u) g(c) \mathrm{d} u\right) \mathrm{d} s<d
$$

where $d=c / 2$. Let $X$ be the Banach space of all continuous real-valued functions $x(t)$ endowed with the norm

$$
\|x\|=\sup _{t \geqslant T}|x(t)|
$$

and with the usual pointwise ordering $\leqslant$. Define a subset $\Omega$ of $X$ as follows:

$$
\Omega=\{x \in X \mid d \leqslant x(t) \leqslant 2 d, t \geqslant T\} .
$$

For any subset $B$ of $\Omega$, it is obvious that inf $B \in \Omega$ and $\sup B \in \Omega$. Let us further define an operator $F: \Omega \rightarrow X$ as follows:

$$
(F x)(t)=d+\int_{T}^{t} a(s) f\left(\beta+\int_{s}^{\infty} b(u) g(x(u)) \mathrm{d} u\right) \mathrm{d} s, t \geqslant T .
$$

The mapping $F$ satisfies the assumptions of Knaster's Fixed Point Theorem [1]: $F$ is non-decreasing and maps $\Omega$ into itself. Indeed, if $x \in \Omega$, then

$$
(F x)(t) \geqslant d, \quad t \geqslant T
$$


and

$$
\begin{aligned}
&(F x)(t) \leqslant d+\int_{T}^{t} a(s) f\left(\int_{T}^{t} b(u) g(2 a) \mathrm{d} u\right) \mathrm{d} s \\
& \quad=d+\int_{T}^{t} a(s) f\left(\int_{T}^{s} b(u) g(c) \mathrm{d} u\right) \mathrm{d} s \leqslant 2 d, \quad t \geqslant T .
\end{aligned}
$$

By Knaster's Fixed Point Theorem [1], we can conclude that there exists an $x \in \Omega$ such that $x=F x$. Set

$$
y(t)=\beta+\int_{t}^{\infty} b(u) g(x(u)) \mathrm{d} u, \quad t \geqslant T
$$

Then

$$
y^{\prime}(t)=-b(t) g(x(t)), \quad \lim _{t \rightarrow \infty} y(t)=\beta>0
$$

and

$$
x^{\prime}(t)=a(t) f(y(t)) .
$$

Hence, $\{x(t), y(t)\}$ is a solution of $(1.1)$ which belongs to $\Omega_{++}(+,+)$. The proof is complete.

By means of similar reasoning used in the proof of Theorem 3.3, we may prove the following two theorems.

Theorem 3.4. Suppose conditions (H1)-(H3) hold and $A\left(t_{0}\right)<\infty$. A necessary and sufficient condition for (1.1) to have a non-oscillatory solution in $\Omega_{++}(+, 0)$ is that

$$
\int_{T}^{\infty} a(s) f\left(\int_{s}^{\infty} b(u) g(c) \mathrm{d} u\right) \mathrm{d} s<\infty
$$

for some $c>0$.

Theorem 3.5. Suppose conditions (H1) $-(H 3)$ hold and $A\left(t_{0}\right)<\infty$. A necessary and sufficient condition for (1.1) to have a non-oscillatory solution in $\Omega_{+_{-}}(+,-)$is that

$$
\int_{T}^{\infty} a(s)\left|f\left(\beta+\int_{s}^{\infty} b(u) g(c) \mathrm{d} u\right)\right| \mathrm{d} s<\infty
$$

for some $c>0$ and $\beta<0$.

Next, we derive two existence criteria for the non-oscillatory solutions in $\Omega_{+-}(+,-\infty)$ and $\Omega_{+-}(0,-\infty)$.

Theorem 3.6. Suppose conditions (H1)-(H3) hold and $A\left(t_{0}\right)<\infty$. Suppose further that $f(-u)=-f(u)$. A necessary and sufficient condition for (1.1) to have a nonoscillatory solution in $\Omega_{+-}(+,-\infty)$ is that

$$
\int_{T}^{\infty} a(s) f\left(\int_{T}^{t} b(u) g(c A(u)) \mathrm{d} u\right) \mathrm{d} s<\infty
$$


for some $c>0$, and

$$
\int_{t_{0}}^{\infty} b(s) \mathrm{d} s=\infty
$$

Proof. Let $\{x(t), y(t)\}$ be a solution of (1.1) such that $\lim _{t \rightarrow \infty} x(t)=\alpha>0$ and $\lim _{t \rightarrow \infty} y(t)=-\infty$. Then there exist two positive constants $c_{1}, c_{2}$ and $T \geqslant t_{0}$ such that $c_{1} \leqslant x(t) \leqslant c_{2}$ for $t \geqslant T$. In view of the first equation of (1.1), we have

$$
x(t)=x(T)+\int_{T}^{t} a(s) f(y(s)) \mathrm{d} s .
$$

Since $\lim _{t \rightarrow \infty} x(t)=\alpha>0$, then

$$
\int_{T}^{\infty} a(s)|f(y(s))| \mathrm{d} s<\infty
$$

Furthermore, we see from the second equation of (1.1) that

$$
y(t)=y(T)-\int_{T}^{t} b(s) g(x(s)) \mathrm{d} s
$$

and

$$
y(T)-\int_{T}^{t} b(s) g\left(c_{2}\right) \mathrm{d} s \leqslant y(t) \leqslant-\int_{T}^{t} b(s) g\left(c_{1}\right) \mathrm{d} s .
$$

Since

$$
|f(y(t))| \geqslant f\left(\int_{T}^{t} b(s) g\left(c_{1}\right) \mathrm{d} s\right)
$$

thus

$$
\int_{T}^{\infty} a(s) f\left(\int_{T}^{s} b(u) g\left(c_{1}\right) \mathrm{d} u\right) \mathrm{d} s<\infty .
$$

On the other hand, in view of (3.7) and the fact that $y(t) \rightarrow-\infty$ as $t \rightarrow \infty$, we have

$$
\int_{T}^{\infty} b(s) \mathrm{d} s=\infty
$$

which implies that (3.6) holds.

Conversely, suppose that (3.5) holds. Choose $T \geqslant t_{0}$ so large that

$$
\int_{T}^{\infty} a(s) f\left(\int_{T}^{s} b(u) g(c) \mathrm{d} u\right) \mathrm{d} s<d,
$$

where $d=c / 2$. Let $X$ be the partly ordered Banach space of all continuous real-valued functions $x(t)$ endowed with the norm

$$
\|x\|=\sup _{t \geqslant T}|x(t)|
$$


and with the usual pointwise ordering $\leqslant$. Define a subset $\Omega$ of $X$ as follows:

$$
\Omega=\{x \in X \mid d \leqslant x(t) \leqslant 2 d, t \geqslant T\} .
$$

For any subset $B$ of $\Omega$, it is obvious that inf $B \in \Omega$ and $\sup B \in \Omega$. Let us further define an operator $F: \Omega \rightarrow X$ as follows:

$$
(F x)(t)=d+\int_{t}^{\infty} a(s) f\left(\int_{T}^{s} b(u) g(x(u)) \mathrm{d} u\right) \mathrm{d} s, \quad t \geqslant T .
$$

The mapping $F$ satisfies the assumptions of Knaster's Fixed Point Theorem [1]: $F$ is non-decreasing and maps $\Omega$ into itself. Indeed, if $x \in \Omega$, then

$$
(F x)(t) \geqslant d, \quad t \geqslant T
$$

and

$$
\begin{aligned}
(F x)(t) \leqslant d+\int_{t}^{\infty} a(s) f & \left(\int_{T}^{s} b(u) g(2 d) \mathrm{d} u\right) \mathrm{d} s \\
& =d+\int_{t}^{\infty} a(s) f\left(\int_{T}^{s} b(u) g(c) \mathrm{d} u\right) \mathrm{d} s \leqslant 2 d, \quad t \geqslant T .
\end{aligned}
$$

By Knaster's Fixed Point Theorem [1] we can conclude that there exists an $x \in \Omega$ such that $x=F x$. Set

$$
y(t)=-\int_{T}^{t} b(u) g(x(u)) \mathrm{d} u, \quad t \geqslant T .
$$

Then

$$
\begin{gathered}
y^{\prime}(t)=-b(t) g(x(t)), \\
-g(2 d) \int_{T}^{t} b(s) \mathrm{d} s \leqslant y(t) \leqslant-g(d) \int_{T}^{t} b(s) \mathrm{d} s,
\end{gathered}
$$

thus, in view of our assumption,

$$
\int_{t_{0}}^{\infty} b(s) \mathrm{d} s=\infty
$$

i.e. (3.6), we see that

$$
x^{\prime}(t)=-a(t) f(-y(t))=a(t) f(y(t)) \text { and } \lim _{t \rightarrow \infty} y(t)=-\infty,
$$

where we have used the assumption that $f(-u)=-f(u)$. Hence, $\{x(t), y(t)\}$ is a solution of (1.1) which belongs to $\Omega_{+-}(+,-\infty)$. The proof is complete.

Theorem 3.7. Suppose conditions $(H 1)-(H 3)$ hold and $A\left(t_{0}\right)<\infty$. If

$$
\int_{T}^{\infty} a(s) f\left(\int_{s}^{\infty} b(u) g(c A(u)) \mathrm{d} u\right) \mathrm{d} s<\infty
$$


for some $c>0$, and

$$
\int_{t_{0}}^{\infty} b(s) g(d A(s)) \mathrm{d} s=\infty
$$

for any $d>0$, then (1.1) has a non-oscillatory solution in $\Omega_{+_{-}}(0,-\infty)$.

The proof is similar to that of Theorem 3.6 and is thus omitted.

Finally, we derive a necessary condition for (1.1) to have a non-oscillatory solution in $\Omega_{+-}(0,-\infty)$.

Theorem 3.8. Suppose conditions (H1)-(H3) hold and $A\left(t_{0}\right)<\infty$. A necessary condition for (1.1) to have a non-oscillatory solution in $\Omega_{+_{-}}(0,-\infty)$ is that

$$
\int_{T}^{\infty} a(s) f\left(\int_{s}^{\infty} b(u) g(c A(u)) \mathrm{d} u\right) \mathrm{d} s<\infty
$$

for some $c>0$, and

$$
\int_{t_{0}}^{\infty} b(s) \mathrm{d} s=\infty
$$

Proof. Let $\{x(t), y(t)\}$ be a solution of equations (1.1) such that $\lim _{t \rightarrow \infty} x(t)=0$ and $\lim _{t \rightarrow \infty} y(t)=-\infty$. Then, by Lemma 3.1, there exist two positive constants $c_{1}, c_{2}$ and $T \geqslant t_{0}$ such that $c_{1} A(t) \leqslant x(t) \leqslant c_{2}$ for $t \geqslant T$. In view of the first equation of (1.1), we have

$$
\infty>x(t)=-\int_{t}^{\infty} a(s) f(y(s)) \mathrm{d} s>0,
$$

That is to say

$$
\int_{t_{0}}^{\infty} a(s)|f(y(s))| \mathrm{d} s<\infty
$$

Furthermore, we see from the second equation of (1.1) that

$$
y(t)=y(T)-\int_{T}^{t} b(s) g(x(s)) \mathrm{d} s,
$$

and

$$
y(T)-\int_{T}^{t} b(s) g\left(c_{2}\right) \mathrm{d} s \leqslant y(t) \leqslant-\int_{T}^{t} b(s) g\left(c_{1} A(s)\right) \mathrm{d} s .
$$

Since

$$
|f(y(t))| \geqslant f\left(\int_{T}^{t} b(s) g\left(c_{1} A(s)\right) \mathrm{d} s\right),
$$

thus

$$
\int_{T}^{\infty} a(s) f\left(\int_{T}^{s} b(u) g\left(c_{1} A(u)\right) \mathrm{d} u\right) \mathrm{d} s<\infty .
$$

On the other hand, in view of (3.10) and $y(t) \rightarrow-\infty$ as $t \rightarrow \infty$, we have

$$
\int_{T}^{\infty} b(s) \mathrm{d} s=\infty
$$

which implies that (3.9) holds. The proof is complete. 
Acknowledgements. W.T.L. was supported by the WNSF of China and the Foundation for University Key Teachers by the Ministry of Education of China.

\section{References}

1. I. GYöRI AND G. LADAS, Oscillation theory of delay differential equations with applications (Clarendon, Oxford, 1991).

2. I. G. E. Kordonis AND CH. G. Philos, On the oscillation of nonlinear two-dimensional differential systems, Proc. Am. Math. Soc. 126 (1998), 1661-1667.

3. T. KUSANO AND M. NAITO, Positive solutions of a class of nonlinear ordinary differential equations, Nonlinear Analysis TMA 12 (1988), 935-942.

4. T. KusAno AND B. Singh, Positive solutions of functional differential equations with singular nonlinear terms, Nonlinear Analysis TMA 8 (1984), 1081-1090.

5. M. K. KwONG AND J. S. W. WONG, Oscillation of Emden-Fowler systems, Diff. Integ. Eqns 1 (1988), 133-141.

6. W. T. LI, Classifications and existence of nonoscillatory solutions of second order nonlinear neutral differential equations, Ann. Polonici Math. LXV (1997), 283-302.

7. W. D. LU, Existence and asymptotic behavior of nonoscillatory solutions of second order nonlinear neutral equations, Acta Math. Sinica 36 (1993), 476-484.

8. D. D. Monzov, Oscillatory properties of solutions of a systems of nonlinear differential equations, Differentsial'nye Uravneniya 9 (1973), 581-583 (in Russian).

9. D. D. Monzov, The oscillation of solutions of a systems of nonlinear differential equations, Mat. Zametki 16 (1974), 571-576 (in Russian).

10. D. D. MoRzov, Oscillatory properties of solutions of a nonlinear Emden-Fowler differential systems, Differentsial'nye Uravneniya 16 (1980), 1980-1984 (in Russian).

11. M. NAITO, Asymptotic behavior of solutions of second order differential equations with integrable coefficients, Trans Am. Math. Soc. 282 (1984), 577-588.

12. M. NAITO, Nonoscillatory solutions of second order differential equations with integrable coefficients, Proc. Am. Math. Soc. 109 (1990), 769-774.

13. M. NAITO, Integral averages and the asymptotic behavior of solutions of second order ordinary differential equations, J. Math. Analysis Appl. 164 (1992), 370-380.

14. J. RUAN, Types and criteria of nonoscillatory solutions of second order linear neutral differential equations, Chinese Ann. Math. A 8 (1987), 114-124. 